Check for updates

Cite this: RSC Adv., 2017, 7, 50403

Received 1st August 2017

Accepted 23rd October 2017

DOI: $10.1039 / c 7 r a 08465 j$

rsc.li/rsc-advances

\section{Controllable wettability and adhesion of superhydrophobic self-assembled surfaces based on a novel azobenzene derivative $\uparrow$}

\author{
Qiongqiong Gao, Liu He, Yajie Li, Xia Ran* and Lijun Guo (D)*
}

Superhydrophobic surfaces with controllable adhesion have received considerable attention due to their potential in numerous applications. Here, we report a controllable adhesion of superhydrophobic surfaces through tuning the self-assembly structure based on a novel Y-shaped molecule AOB-Y8, consisting of azobenzene groups, 1,3,4-oxadiazole moieties, and three octyl chains. The surface hydrophobicity of $\mathrm{AOB}-\mathrm{Y} 8$ films was successfully regulated by tuning the assembly morphologies through changing the composition of $\mathrm{CHCl}_{3} / \mathrm{CH}_{3} \mathrm{CN}$ mixed solvents, concentration and temperature. Morphological studies of the film prepared from $50: 50 \mathrm{CHCl}_{3} / \mathrm{CH}_{3} \mathrm{CN}$ solvents revealed that the selfassembled hierarchical, flower-like structure constructs a lot of grooves to trap the surrounding air, generating the superhydrophobic effect at room temperature. Particularly, the surface adhesion of this superhydrophobic film was further regulated from a low level to a very high level by simply changing the concentration of AOB-Y8 in mixed solvents. Meanwhile, the AOB-Y8 self-assembled surfaces showed an excellent chemical resistance to acid and alkali, which is suitable for applications in many environmental conditions. As examples, the tunable adhesive superhydrophobic AOB-Y 8 surfaces demonstrated good features to be used in selective transportation of droplets, self-cleaning and droplet-based microreactors to quantitatively detect $\mathrm{NaOH}$ and $\mathrm{FeCl}_{3}$.

\section{Introduction}

Hydrophobic surfaces with tunable wettability and adhesion have received considerable attention due to their great potential for applications in many fields, such as self-cleaning, ${ }^{1}$ antisnow/fog, ${ }^{2}$ antisticking, ${ }^{3}$ anticorrosion, ${ }^{4}$ oil/water separation, ${ }^{5}$ and microdroplet transportation. ${ }^{6}$ Besides biomimetic methods, the physicochemical wetting concept is in part feasible for fabricating superhydrophobic surfaces by combining a low surface energy material with surface roughness/morphology at the micro/nano scale. ${ }^{7-9}$ Generally, superhydrophobic surfaces can be normally classified into two types: low adhesion and high adhesion to water. Low-adhesion superhydrophobic surfaces are usually inspired by biological organisms, the self-cleaning lotus leaf is the typical example. ${ }^{\mathbf{1 0}}$ On the other hand, the high-adhesion superhydrophobic surface is inspired by the gecko's attachment system and rose petals. ${ }^{11}$ On these surfaces, water drop shows a static water contact angles (CA) larger than $150^{\circ}$, but the drop is pinned on the surfaces at any titled angles. ${ }^{12}$ It has been previously demonstrated that liquid adhesion to solid surface can be

Institute of Micro/Nano Photonic Materials and Application, Henan University, Kaifeng 475004, China. E-mail: ranxia@henu.edu.cn; juneguo@henu.edu.cn

$\uparrow$ Electronic supplementary information (ESI) available. See DOI: $10.1039 / \mathrm{c} 7 \mathrm{ra08465j}$ regulated by either tuning the surface chemical composition or tailoring the morphology. ${ }^{\mathbf{1 3 - 1 8}}$ For example, Guittard and coworkers obtained highly hydrophobic surfaces with various sticking behaviors from electro-deposition of poly(3,4-bis(alkoxy)thiophene)s. ${ }^{19} \mathrm{Li}$ group successfully fabricated tunable adhesive superhydrophobic $\mathrm{ZnO}$ surfaces by spraying $\mathrm{ZnO}$ nanoparticle suspensions onto desired substrates. ${ }^{13}$ Jiang group reported on a versatile strategy to reversibly control the mobility of spherical microdroplets of water on the same surface from rollable to pinned by temperature. ${ }^{20}$

Whether water droplets are pinned on (high adhesion) or rolled off (low adhesion) a particular superhydrophobic surface depends on the distinct contact modes: the Wenzel state, the metastable state, and the Cassie state. ${ }^{21,22}$ In the Wenzel state, ${ }^{23}$ the water droplet completely fills the grooves of the rough surface at the point of contact. Thus, the Wenzel state exhibits an extremely high adhesive property. Conversely, in the Cassie state, ${ }^{24}$ the water droplet contacts the top of asperities with air trapped under the liquid. In this state, the water droplet can easily roll off the surface with extremely low adhesion. Eclectically, in the metastable state, a water droplet partially wets the roughness features with air trapped in the rest of valleys, thus the adhesion of the surface is between that of the above two states and the water droplet rolls off with the surface significantly tilted. ${ }^{25}$ So far, there is still a practical interest to produce highly hydrophobic surfaces with various adhesive properties. 


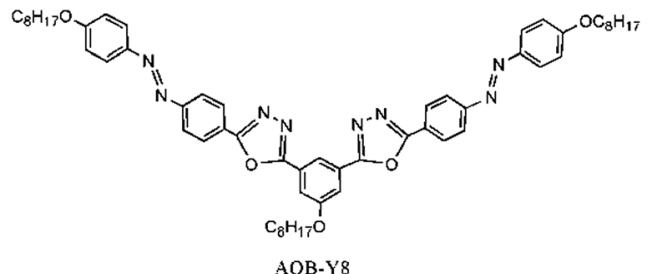

Fig. 1 Molecular structure of AOB-Y8.

However, previous reports mostly focused on the construction of superhydrophobic and tunable adhesive surface with polymer molecules or inorganic materials, ${ }^{26-39}$ in spite of the fact that small organic molecules have well-defined molecular structures and are easy to synthesize and modify. When assembling surfaces from organic molecule, molecular interactions such as hydrogen bonding, $\pi-\pi$ stacking, and van der Waals interaction play a key role in determining the ultimate structures and surface properties. Solvent mediated balancing of these interactions is an effective way to construct various film morphologies with different surface wettability and adhesion. To regulate surface wettability and adhesion through solvent and concentration tuning, we designed and synthesized a novel Y-shaped compound AOB-Y8 containing azobenzene groups, 1,3,4-oxadiazole moieties, and three octyl chains (Fig. 1). We then demonstrated the potential applications of this novel azobenzene derivative by fabricating tunable adhesive superhydrophobic surfaces that could be used as a droplet transporter, self-cleaner and droplet-based microreactor.

\section{Results and discussion}

AOB-Y8 is soluble in non-polar and moderate polar solvents but insoluble in strong polar solvents. A facile and versatile approach of regulating self-assembly structure is to introduce a second liquid, and the introduced liquid component is usually miscible with the original solvent. In order to regulate the selfassembly structure with different surface wettability and adhesion of AOB-Y8, we first investigated the self-assembly behavior of AOB-Y8 in chloroform-acetonitrile mixed solvents. As shown in Fig. 2a, AOB-Y8 in dilute solution $\left(1 \times 10^{-5} \mathrm{M}\right)$ shows an intense absorption around $374 \mathrm{~nm}$ in chloroform, as well as
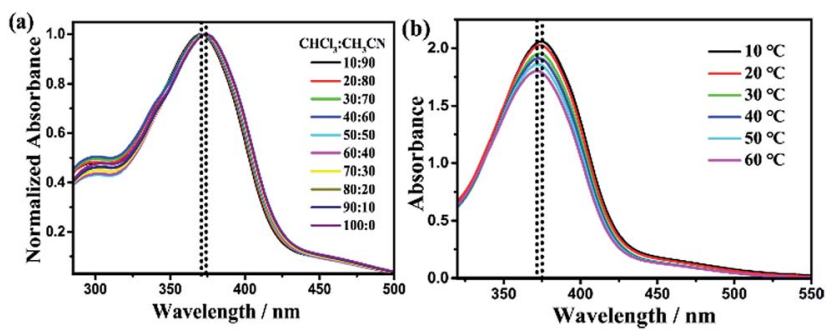

Fig. 2 (a) Normalized UV/Vis absorption spectra of AOB-Y8 in $\mathrm{CHCl}_{3}$ : $\mathrm{CH}_{3} \mathrm{CN}$ solution $\left(1 \times 10^{-5} \mathrm{M}\right)$ with different solvent ratio. (b) Temperature-dependent UV/Vis absorption spectra of $\mathrm{AOB}-\mathrm{Y} 8$ in $50: 50 \mathrm{CHCl}_{3} / \mathrm{CH}_{3} \mathrm{CN}\left(5 \times 10^{-4} \mathrm{M}\right)$. a weak band around $450 \mathrm{~nm}$. The large absorption band at shorter wavelength belongs to a $\pi-\pi^{*}$ type absorption of the trans-azobenzene moieties. The position of the absorbance maxima is slightly dependent on the compositions of $\mathrm{CHCl}_{3}$ / $\mathrm{CH}_{3} \mathrm{CN}$ mixed solvents, with a total red-shift from $371 \mathrm{~nm}$ to $374 \mathrm{~nm}$ when changing from $10: 90 \mathrm{CHCl}_{3} / \mathrm{CH}_{3} \mathrm{CN}$ to $90: 10$ $\mathrm{CHCl}_{3} / \mathrm{CH}_{3} \mathrm{CN}$. The temperature and concentration dependence of absorption spectroscopies were also employed to investigate the effects of molecular arrangements on self-assembly behaviours. For the $5 \times 10^{-5} \mathrm{M}$ of AOB-Y8 in $50: 50 \mathrm{CHCl}_{3} / \mathrm{CH}_{3} \mathrm{CN}$, the $\pi-\pi^{*}$ absorption maximum of azobenzene group locates around $372 \mathrm{~nm}$ at $60^{\circ} \mathrm{C}$ and almost stays at the same position at $10{ }^{\circ} \mathrm{C}$ in the diluted AOB-Y8 solution (Fig. S1 $\dagger$ ). Fig. $2 \mathrm{~b}$ shows the temperature-dependent UV-Vis absorption spectra of $5 \times$ $10^{-4} \mathrm{M}$ of AOB-Y8 in $50: 50 \mathrm{CHCl}_{3} / \mathrm{CH}_{3} \mathrm{CN}$ mixed solvents, which clearly demonstrates the existence of aggregation state at higher concentration. Specifically, the $\pi-\pi^{*}$ absorption maximum of azobenzene group locates around $372 \mathrm{~nm}$ at $60{ }^{\circ} \mathrm{C}$ and red-shifts to $375 \mathrm{~nm}$ at $10{ }^{\circ} \mathrm{C}$, indicating the role of J-type molecular arrangement through $\pi-\pi$ interactions in regulating the self-assembly behavior of AOB-Y8 molecule in mixed solvent. ${ }^{40,41}$

The AOB-Y8 surfaces were simply fabricated by casting a small drop of AOB-Y8 solution in $\mathrm{CHCl}_{3} / \mathrm{CH}_{3} \mathrm{CN}$ mixed solvents with different composition onto silicon plates at room temperature. Fig. 3 shows the scanning electron microscopy (SEM) images and water contact angle (CA) photographs of drop-cast AOB-Y8 surfaces, clearly demonstrating a strong dependence on the nature of solvents. With a composition of $10 \% \mathrm{CH}_{3} \mathrm{CN}$ (Fig. 3a), the morphology of assembled surface consists of flexible root-like fibres with an average width of $540 \mathrm{~nm}$ and length of a few micrometers. With the $\mathrm{CH}_{3} \mathrm{CN}$ component increased to $50 \%$ and $60 \%$, we observed a more flexible and dense flower-like nanowire array with an average width of $360 \mathrm{~nm}$ (Fig. 3b and c). These self-assembled hierarchical flower-like structures can generate numerous grooves to
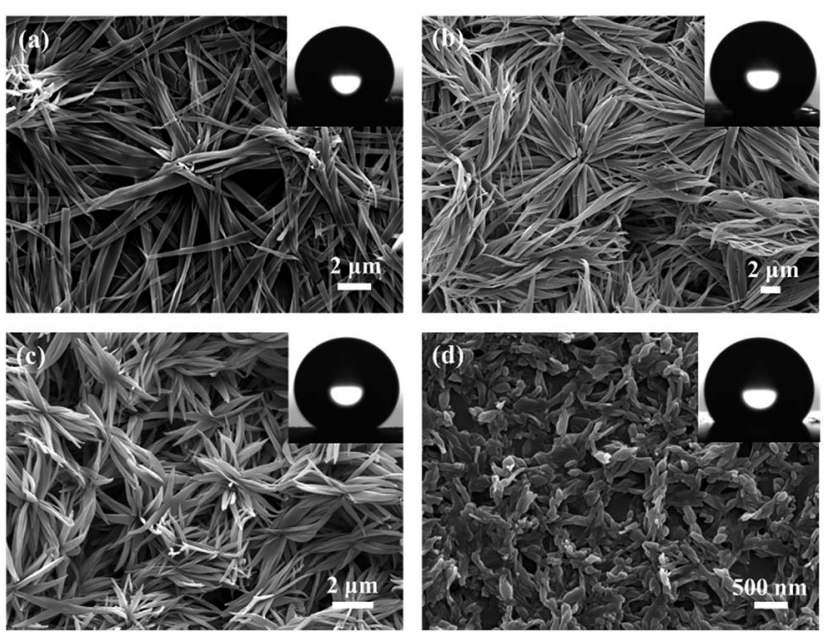

Fig. 3 SEM images and water CA photographs of AOB-Y8 surfaces prepared from $1 \mathrm{mg} \mathrm{mL}^{-1}$ in $\mathrm{CHCl}_{3}: \mathrm{CH}_{3} \mathrm{CN}$ solution with different solvent ratio: (a) $90: 10$, (b) $50: 50$, (c) $40: 60$, (d) $10: 90$ on silicon substrates at $25^{\circ} \mathrm{C}$. 
trap the surrounding air, thus leading to a superhydrophobic effect. ${ }^{42-44}$ However, when the $\mathrm{CH}_{3} \mathrm{CN}$ was increased to $90 \%$ (Fig. 3d), the floral structures disappeared thoroughly and transformed into short sticks with the average diameter of $100 \mathrm{~nm}$ and length of several hundred nanometers. These regulated morphologies of AOB-Y8 surfaces by $\mathrm{CHCl}_{3} / \mathrm{CH}_{3} \mathrm{CN}$ mixed solvents are expected to be closely associated with the surface wettability. As shown in Fig. S2, $\dagger$ with the increase of $\mathrm{CH}_{3} \mathrm{CN}$ composition, the corresponding $\mathrm{CA}$ of water droplets on the as-prepared surfaces first increases and then decreases, and reaches a superhydrophobic CA of $151.2^{\circ}$ for $\mathrm{CHCl}_{3}: \mathrm{CH}_{3} \mathrm{CN}=$ $50: 50$. Obviously, solvent is responsible for the changes in structure and wettability of these self-assembled AOB-Y8 surfaces.

In an attempt to dissect the factors related to the changes in the morphologies and wettability, we have calculated the Hansen solubility parameters (HSPs) of the solvent mixtures and discussed the correlation between the HSPs and the morphology of AOB-Y8. HSPs have been successfully used for several decades to select solvents for coating materials in the field of polymer science.$^{45}$ In this approach, the cohesive energy density is decomposed into three contributions: dispersive interactions $\left(\delta_{\mathrm{d}}\right)$, polar interactions $\left(\delta_{\mathrm{p}}\right)$ and hydrogen bonds $\left(\delta_{\mathrm{h}}\right)$. Fig. 4 gives the HSPs of different $\mathrm{CHCl}_{3}: \mathrm{CH}_{3} \mathrm{CN}$ compositions, and the values for the HSPs of single solvents can be found in the literature. ${ }^{45,46}$ It can be seen that the value of $\delta_{\mathrm{p}}$ increases with the increasing of $\mathrm{CH}_{3} \mathrm{CN}$ composition, but the value of $\delta_{\mathrm{d}}$ and $\delta_{\mathrm{h}}$ remain almost invariant. When the value of $\delta_{\mathrm{p}}$ is 4.59 , the morphology of assembled surface consists of flexible root-like fibers with the corresponding CA of $133.4^{\circ}$ (Fig. S2 $\dagger$ ). With the value of $\delta_{\mathrm{p}}$ increases to 10.55 and 12.04, we observed the flower-like nanowire array with the superhydrophobic CA (Fig. S2 $\dagger$ ). However, when the value of $\delta_{\mathrm{p}}$ is increased to 16.51, the floral structures disappears thoroughly with the corresponding CA of $129.8^{\circ}$. Therefore, the major contributor to the morphology and wettability changes can be attributed to the polar interactions parameter, $\delta_{\mathrm{p}}$. Meanwhile, considering the fact that the boing point of $\mathrm{CH}_{3} \mathrm{CN}$ is higher than that of $\mathrm{CHCl}_{3}$, it can be deduced that the increasing proportion of $\mathrm{CH}_{3} \mathrm{CN}$ slows down the evaporation rate of mixed solvent and there is

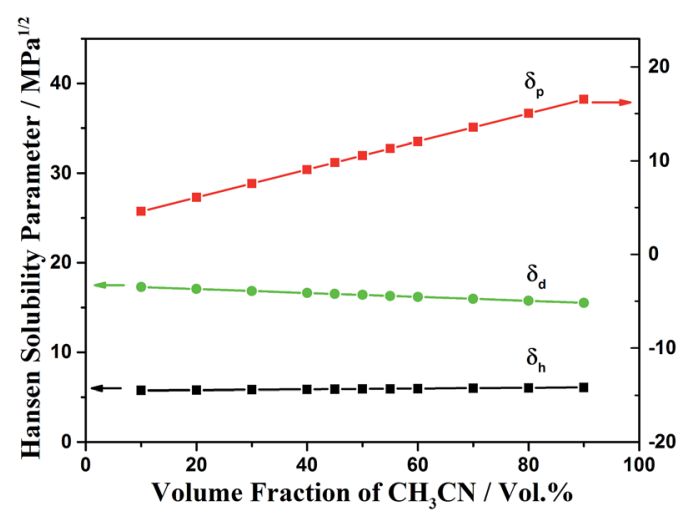

Fig. 4 Hansen solubility parameters $\left(\mathrm{MPa}^{1 / 2}\right)$ of different $\mathrm{CHCl}_{3}: \mathrm{CH}_{3} \mathrm{CN}$ compositions. more time to grow the crystallites of AOB-Y8, thus resulting in the obtained different morphologies of AOB-Y8 from different compositions of $\mathrm{CHCl}_{3}: \mathrm{CH}_{3} \mathrm{CN}$ mixed solvent.

The XRD patterns of AOB-Y8 surfaces prepared from $3 \mathrm{mg} \mathrm{mL}$ in different $\mathrm{CHCl}_{3}: \mathrm{CH}_{3} \mathrm{CN}$ compositions are compared in Fig. $\mathrm{S} 3, \dagger$ in which the corresponding $d$ values of diffraction peaks were calculated from the Bragg equation. The patterns of AOBY8 surfaces prepared from $\mathrm{CHCl}_{3}: \mathrm{CH}_{3} \mathrm{CN}=50: 50$ and $\mathrm{CHCl}_{3}: \mathrm{CH}_{3} \mathrm{CN}=40: 60$ display a series of sharper diffractions than that of AOB-Y8 surfaces prepared from $\mathrm{CHCl}_{3}: \mathrm{CH}_{3} \mathrm{CN}=$ $90: 10$ and $\mathrm{CHCl}_{3}: \mathrm{CH}_{3} \mathrm{CN}=10: 90$, indicating the existence of a crystal-like ordered structure in the superhydrophobic surfaces. Specifically, the XRD data of AOB-Y8 surfaces prepared from $\mathrm{CHCl}_{3}: \mathrm{CH}_{3} \mathrm{CN}=50: 50$ demonstrate a series of sharp diffractions with the main peaks centred at $2 \theta=2.03^{\circ}(d=$ $43.56 \AA), 2 \theta=4.07^{\circ}(d=21.71 \AA), 2 \theta=6.26^{\circ}(d=14.11 \AA)$ and $2 \theta$ $=8.67^{\circ}(d=10.12 \AA)$ in the low-angle region. The $d$-spacing ratio of $1: 1 / 2: 1 / 3: 1 / 4$ in the low-angle regions suggests a lamellar structure and an interlayer distance of $43.56 \AA^{47,48}$ This distance is smaller than the calculated molecular length of AOB-Y8 (52.13 $\AA$, estimated by the DFT molecular modelling), implying a possible tilt or interdigitated packing arrangement of AOB-Y8 molecule in layers. The existence of other three peaks centred at $2 \theta=5.01,9.95$ and $14.93^{\circ}$ corresponding to $d$-spacings of $17.62,8.88$ and $5.93 \AA$, respectively, indicates that other types of aggregates also exist in the superhydrophobic surfaces ${ }^{49}$ The AOB-Y8 surfaces prepared from $\mathrm{CHCl}_{3}: \mathrm{CH}_{3} \mathrm{CN}$ $=40: 60$ shows the similar spectral features, and thus indicates a similar packing mode of AOB-Y8.

Furthermore, the temperature control during the casting process might also affect the evaporation, condensation, surface tension, viscosity of the solution and solubility. By adjusting the initial temperature of substrates, we found the temperature imposes an important effect on the morphologies of the AOB-Y8 surfaces. For comparison of the well-developed flat nanowires structure prepared from $\mathrm{CHCl}_{3}: \mathrm{CH}_{3} \mathrm{CN}=$ $50: 50$ on silicon substrates at $25^{\circ} \mathrm{C}$ and $1 \mathrm{mg} \mathrm{mL}^{-1}$ (Fig. 3b), the SEM images from the solution of AOB-Y8 at other two different temperatures $\left(5{ }^{\circ} \mathrm{C}\right.$ and $\left.60^{\circ} \mathrm{C}\right)$ are presented in Fig. 5 . At $5{ }^{\circ} \mathrm{C}$, the surface is composed of more flexible nanowires with the average width of $340 \mathrm{~nm}$ (Fig. 5a), the corresponding CA of $148.1^{\circ}$. However, when the substrate temperature was set to $60{ }^{\circ} \mathrm{C}$, we observed a surface composed of an acute and flowerlike sheet array with an average thickness of $220 \mathrm{~nm}$ (Fig. 5b),

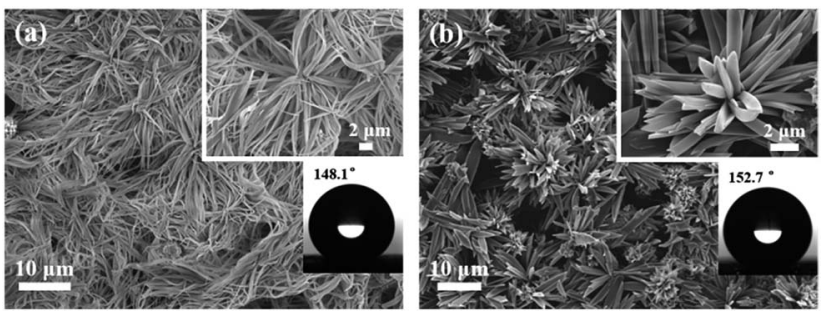

Fig. 5 SEM images of $A O B-Y 8$ surfaces prepared from $1 \mathrm{mg} \mathrm{mL}^{-1}$ in $50: 50 \mathrm{CHCl}_{3}: \mathrm{CH}_{3} \mathrm{CN}$ solution on silicon substrates at (a) $5^{\circ} \mathrm{C}$ and (b) $60{ }^{\circ} \mathrm{C}$. 
and the CA reaches a superhydrophobic value of $152.7^{\circ}$. These observations confirm that temperature is a critical determinant for forming the superhydrophobic surfaces and their assembly morphology.

The surface adhesivity is a vitally important parameter among various surface properties, reflecting the ability to deliver liquid on surface. Therefore, superhydrophobic surfaces with tunable water adhesion have become a new focus due to their potential applications in liquid transportation, biochemical separation, and microfluid systems. ${ }^{8-12}$ Besides being superhydrophobic, the self-assembly surface of AOB-Y8 from $\mathrm{CHCl}_{3}: \mathrm{CH}_{3} \mathrm{CN}=50: 50$ mixed solvents also demonstrates a strong adhesive force to hold water droplet even when the surface is tuned upside down (Fig. S4†). Interestingly, we regulated the water adhesion of these superhydrophobic surfaces by simply changing the concentration of AOB-Y8 in $50: 50 \mathrm{CHCl}_{3} / \mathrm{CH}_{3} \mathrm{CN}$ mixed solvents. With the concentration increased from $1 \mathrm{mg} \mathrm{mL}^{-1}$ to $6 \mathrm{mg} \mathrm{mL}^{-1}$, as shown in Fig. 6 , the water CAs increased from $151 \pm 0.6^{\circ}$ to $159 \pm 0.4^{\circ}$, and the corresponding water sliding angle decreased from $90^{\circ}$ to $62 \pm 1^{\circ}$, $31 \pm 2^{\circ}, 19 \pm 1^{\circ}, 18 \pm 3^{\circ}$, and $15 \pm 3^{\circ}$, respectively. Concomitantly, the contact angle hysteresis (CAH) of water droplet demonstrates a dependence on the concentration of AOB-Y8 as well (Table $\mathrm{S} 1 \dagger)$. Quantitatively, the adhesive force of these as-prepared superhydrophobic surfaces was further characterized by using a high-sensitive microelectromechanical balance system. Fig. 7a exhibits a typical adhesive force measuring process, and Fig. 7b shows the adhesive force being dependent on AOB-Y8 concentration in $50: 50 \mathrm{CHCl}_{3} / \mathrm{CH}_{3} \mathrm{CN}$ mixed solvents. As a result, the adhesive force of superhydrophobic surfaces can be tuned from a low level of $13 \mu \mathrm{N}$ to a very high level of $85 \mu \mathrm{N}$ by decreasing the concentration of AOB-Y8 from $5 \mathrm{mg} \mathrm{mL}^{-1}$ to $1 \mathrm{mg} \mathrm{mL}^{-1}$ in mixed solvents.

To describe the correlation between surface adhesion and self-assembly structure, we carried out morphology measurements of the AOB-Y8 surfaces from different concentrations in mixed solvent. Fig. 8 and $\mathrm{S} 5 \dagger$ show the SEM cross-sectional and planar view of AOB-Y8 surfaces from $50: 50 \mathrm{CHCl}_{3} / \mathrm{CH}_{3} \mathrm{CN}$ solutions with the concentration ranging from $1 \mathrm{mg} \mathrm{mL}^{-1}$ to $5 \mathrm{mg} \mathrm{mL}{ }^{-1}$. In the case of $1 \mathrm{mg} \mathrm{mL}^{-1}$, the surface morphology is microscopically composed of well-developed flat nanowires with the average width of $360 \mathrm{~nm}$ and length of a few micrometers (Fig. S5a $\dagger$ ). Macroscopically, these nanowires are constructed into a hill-like morphology consisting of $18 \pm 0.1$
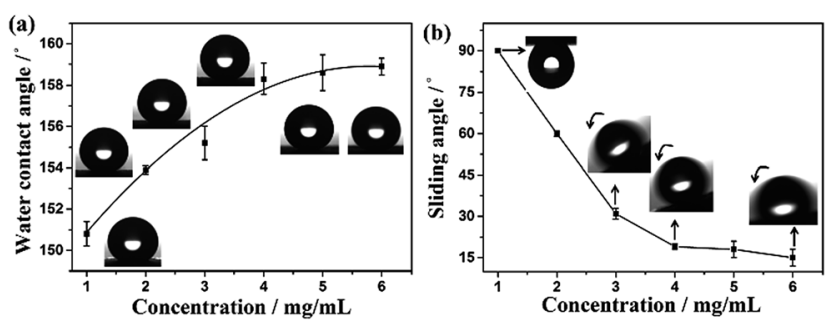

Fig. 6 Dependences of (a) water contact angle and (b) sliding angle on the concentration of $A O B-Y 8$ in $50: 50 \mathrm{CHCl}_{3} / \mathrm{CH}_{3} \mathrm{CN}$ mixed solvents. Insets are the shapes of water droplets $(4 \mu \mathrm{L})$ on $\mathrm{AOB}-\mathrm{Y} 8$ surface. (a)

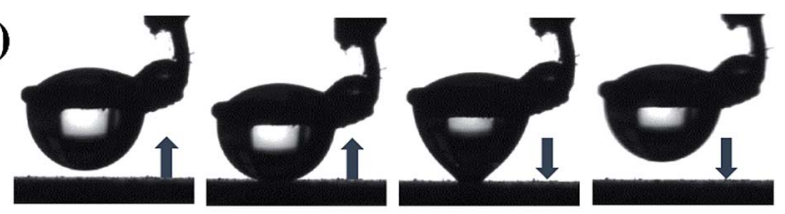

(b)

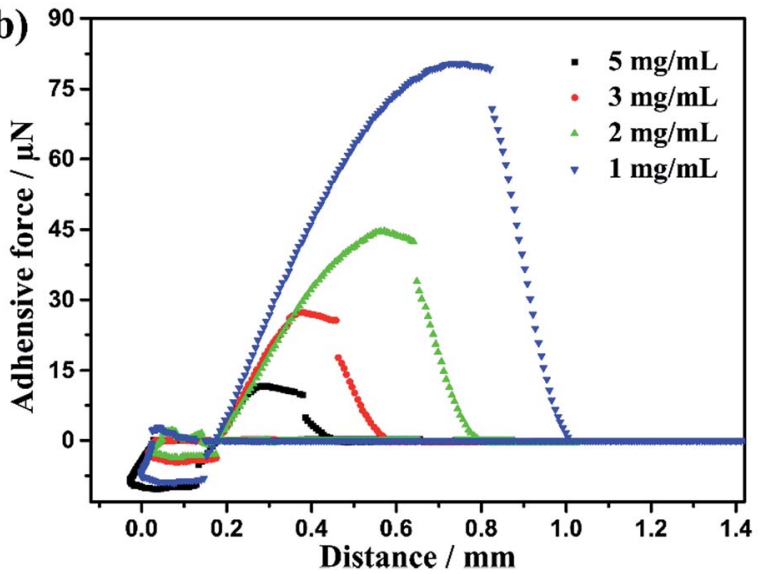

Fig. 7 Dynamical adhesion measurement on the as-prepared surface: (a) CCD images for a typical measurement process. (b) Force-distance curves on the superhydrophobic surfaces prepared with the AOB-Y8 concentrations of $1,2,3$ and $5 \mathrm{mg} \mathrm{mL}^{-1}$ in $50: 50 \mathrm{CHCl}_{3}: \mathrm{CH}_{3} \mathrm{CN}$ mixture on silicon substrates at room temperature, respectively.

$\mu \mathrm{m}$ tower peaks and $10 \pm 0.1 \mu \mathrm{m}$ deep valleys in the film (Fig. 8a). With the increase of AOB-Y8 concentration to $5 \mathrm{mg}$ $\mathrm{mL}^{-1}$, a flower-like morphology with a diameter of 10-18 $\mu \mathrm{m}$ and the petals upright like thorns of $6-10 \mu \mathrm{m}$ in length is formed (Fig. S5d $\dagger$ and 8c). This observed difference in adhesion of superhydrophobic surfaces can be well described using Cassie's theory and Wenzel's theory, ${ }^{7}$ respectively. In the Cassie state, the contact angle on the pincushion-like surface can be described by the Cassie's equation:
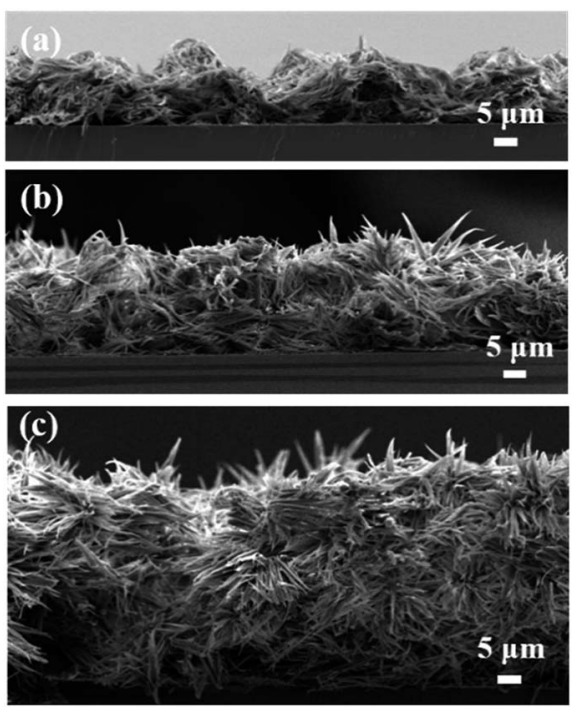

Fig. 8 Dependence of cross-sectional view of $A O B-Y 8$ surfaces prepared from different concentrations in $50: 50 \mathrm{CHCl}_{3} / \mathrm{CH}_{3} \mathrm{CN}$ solvents at $25^{\circ} \mathrm{C}$ : (a) $1 \mathrm{mg} \mathrm{mL}^{-1}$, (b) $3 \mathrm{mg} \mathrm{mL}^{-1}$ and (c) $5 \mathrm{mg} \mathrm{mL}^{-1}$. 


$$
\cos \vartheta=f_{1} \cos \vartheta_{\text {flat }}-f_{2}
$$

where $\vartheta$ and $\vartheta_{\text {flat }}$ are the static water contact angle on rough and plane surfaces respectively, the $f_{1}$ and $f_{2}$ are the area fraction of the solid surface and of the air in the interspaces among the microstructures, respectively (i.e., $f_{1}+f_{2}=1$ ). The $\vartheta_{\text {flat }}$ of surfaces with different modification conditions is almost unchanging, so when the contact angle increases, the $f_{1}$ would decrease. This means the friction force between the surface and the liquid would decrease and lead to the decrease of the sliding angle and the contact angle hysteresis (CAH) of water droplet (Table S1†). In such case, water droplets basically float on the pincushion-like surface, and the contact area between surface and droplet is too small to wet the space among nanofibers, thus resulting in a small surface adhesion (Fig. 9). With the transformation of surface morphology into hill-like structure, the contact state is switched into the Wenzel model, and the adhesion becomes strong enough to firmly pin water droplet at various tilted angles (Fig. $\mathrm{S} 4 \dagger$ ). In the compromised situation, the droplet partially wets the contact area where the hill-like morphology is not completely transformed into a pincushionlike structure (Fig. 8b and S5c $\dagger$ ), so the adhesion locates at the metastable state between Cassis state and Wenzel state.

The environmental adaptability of superhydrophobic surface is an important factor for its practical application. The AOB-Y8 superhydrophobic surfaces with tuneable adhesion exhibit a good acid/base-resisting property. The contact angle or superhydrophobicity changes little when the water $\mathrm{pH}$ varies from 1 to 14 , indicating chemical stability (Fig. S6†). Furthermore, the as-prepared AOB-Y8 surfaces can retain such wettability even after 3 months without special protection, demonstrating a good durability as well.

The tuneable surface adhesion and selective microdroplet transportation of AOB-Y8 superhydrophobic surfaces can be applied as droplet-based microreactor. ${ }^{50-52}$ As an example, we performed the selective and step-by-step transportation of microdroplets towards the quantitative detection of $\mathrm{NaOH}$ and $\mathrm{FeCl}_{3}$ in a microreactor. In Fig. 10a, two droplets were placed on the AOB-Y8 superhydrophobic surface with low adhesion to water: left, $4 \mu \mathrm{L}$ of $0.3 \mathrm{~mol} \mathrm{~L}^{-1} \mathrm{CuCl}_{2}$; right, $8 \mu \mathrm{L}$ of $0.6 \mathrm{~mol} \mathrm{~L}^{-1}$ $\mathrm{NaOH}$. These two water droplets were contacted with the medium adhesive AOB-Y8 superhydrophobic surface, and only the $\mathrm{CuCl}_{2}$ droplet was captured by the surface and the $\mathrm{NaOH}$ droplet stayed in situ (Fig. 10b and c). Subsequently, the $\mathrm{CuCl}_{2}$ droplet was transported to contact with another $4 \mu \mathrm{L}$ of

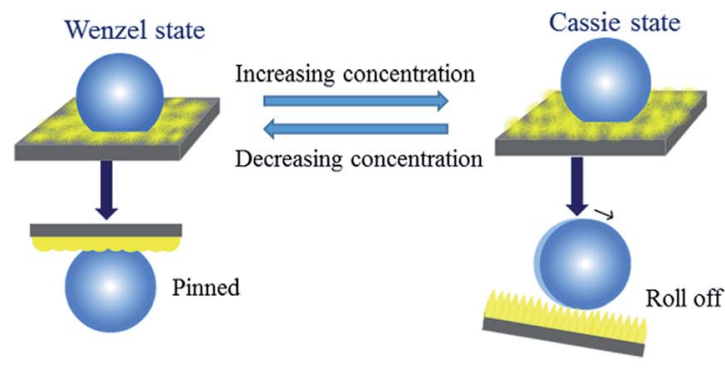

Fig. 9 Schematic illustration of liquid-solid contact modes.
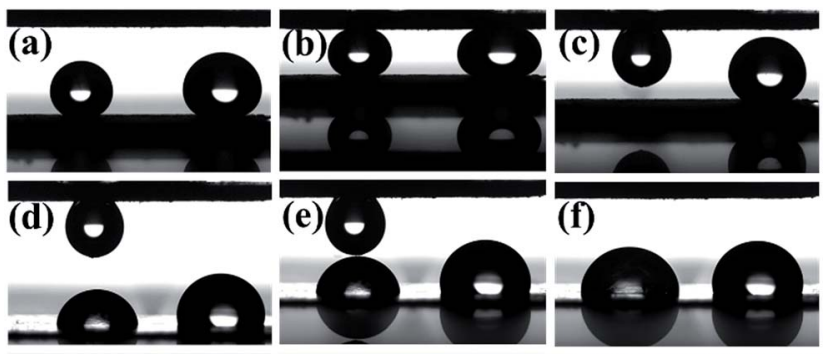

(f)
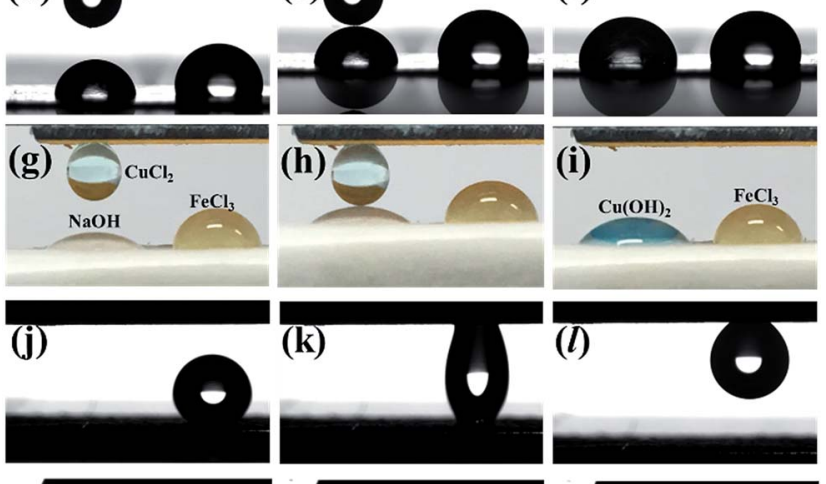

(k)

(i)
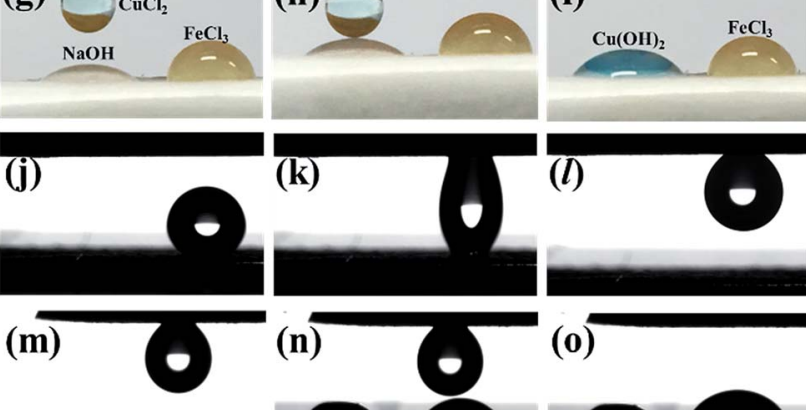

(l)

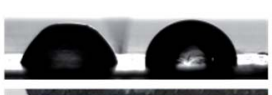

(n)
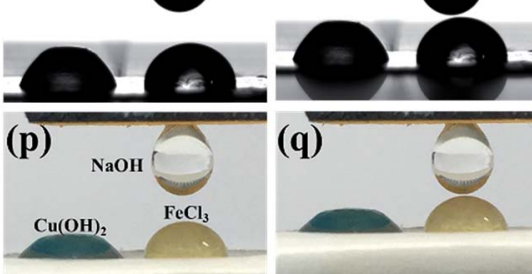

(o)

Fig. 10 Tunable adhesive superhydrophobic surface used as a microreactor to detect $\mathrm{NaOH}$ and $\mathrm{FeCl}_{3}$.

$0.6 \mathrm{~mol} \mathrm{~L}^{-1} \mathrm{NaOH}$ droplet on a silicon paper (Fig. 10d, e, g and h). These two droplets coalesced and produced the blue $\mathrm{Cu}(\mathrm{OH})_{2}$ after reaction (Fig. $10 \mathrm{f}$ and i). Similarly, the $8 \mu \mathrm{L}$ of $0.6 \mathrm{~mol} \mathrm{~L}^{-1}$ $\mathrm{NaOH}$ droplet was transported to the high adhesion superhydrophobic surface (Fig. 10j-l), and reacted with $8 \mu \mathrm{L}$ of $0.2 \mathrm{~mol} \mathrm{~L}^{-1} \mathrm{FeCl}_{3}$ droplet to produce reddish-brown $\mathrm{Fe}(\mathrm{OH})_{3}$ in a microreactor (Fig. 10m-r). Meanwhile, these superhydrophobic surfaces with low water adhesion also demonstrate a good self-cleaning effect or antifouling ability (Fig. S7 $\dagger$ ), although the dusts in our experiments are far more than those in natural environments.

\section{Experimental}

\section{Synthesis}

The compound AOB-Y8 was synthesized according to the routes shown in Scheme S1. $\dagger$ 4-(4'-Octanoxyphenyl) azobenzoic acid was prepared according to the literature. ${ }^{53,54} 4$-(4'-Octanoxyphenyl) azobenzoic acid (3.2 g, $0.009 \mathrm{~mol})$ and thionyl chloride $(50 \mathrm{~mL})$ were refluxed for $10 \mathrm{~h}$, and the product of (4'-octanoxyphenyl) azobenzoic acid chloride was collected after removing unreacted thionyl chloride. The purified (4'-octanoxyphenyl) azobenzoic acid chloride was dissolved in tetrahydrofuran, and then 5octanoxyphenyl-1,3-benzenedicarboxylic acid dihydrazide (A) ${ }^{55}$ was added slowly. The resulting mixture was stirred for $10 \mathrm{~h}$, and then the reaction mixture was poured into an excess of ice water. 
The precipitant was recrystallized from $80: 20$ tetrahydrofuran/ ethanol mixed solvents to obtain BNB-Y8.

${ }^{1} \mathrm{H}$ NMR (300 MHz, DMSO-d6), (ppm, from TMS): 10.75 (s, $4 \mathrm{H}), 8.19-8.07$ (m, 5H), 8.03-7.87 (m, 8H), 7.69 (s, 2H), 7.21-7.11 $(\mathrm{d}, 4 \mathrm{H}, J=8.04), 4.32-3.92(\mathrm{~m}, 6 \mathrm{H}), 1.91-1.65(\mathrm{~m}, 6 \mathrm{H}), 1.57-1.15$ (m, 30H), 0.98-0.76 (m, 9H).

FT-IR (KBr, pellet, $\mathrm{cm}^{-1}$ ): 3206, 2926, 2856, 1669, 1644, 1598, 1502, 1468, 1394, 1303, 1252, 1184, 1141, 1048, 860, 837, 768, 729, 679 .

Elemental analysis: calculated for $\mathrm{C}_{58} \mathrm{H}_{74} \mathrm{~N}_{8} \mathrm{O}_{7}$ (\%): C, 69.99; H, 7.49; N, 11.26. Found: C, 68.75; H, 7.56; N, 11.18.

The purified BNB-Y8 was dissolved in phosphorous oxychloride $\left(\mathrm{POCl}_{3}\right)$ and refluxed for approximately $30 \mathrm{~h}$. The excess $\mathrm{POCl}_{3}$ was removed through distillation and the residue was slowly added to ice-water. After removal of the solvent under reduced pressure, the final product AOB-Y8 was purified by recrystallization from $20: 80$ tetrahydrofuran/ethanol mixed solvents for further NMR, FT-IR spectroscopy, and elemental analysis.

${ }^{1} \mathrm{H}$ NMR (300 MHz, $\left.\mathrm{CDCl}_{3}-d 6\right)$, (ppm, from TMS): 8.47 (s, 1H), 8.37-8.28 (d, $4 \mathrm{H}, J=7.89$ ), 8.1-8.01 (d, $4 \mathrm{H}, J=7.26$ ), 8-7.92 (d, $4 \mathrm{H}, J=7.06$ ), 7.88 (s, 2H), 7.08-6.97 (d, $4 \mathrm{H}, J=7.7), 4.24-4.01$ $(\mathrm{m}, 6 \mathrm{H}), 1.95-1.76(\mathrm{~m}, 6 \mathrm{H}), 1.69-1.2(\mathrm{~m}, 30 \mathrm{H}), 0.97-0.84$ $(\mathrm{m}, 9 \mathrm{H})$.

FT-IR (KBr, pellet, $\mathrm{cm}^{-1}$ ): 3068, 2925, 2858, 1598, 1545, 1495 , 1471, 1404, 1365, 1303, 1251, 1138, 1076, 1038, 1013, 968, 887, 847, 783, 750, 723, 686, 634, 586, 548, 507, 478.

Elemental analysis: calculated for $\mathrm{C}_{58} \mathrm{H}_{70} \mathrm{~N}_{8} \mathrm{O}_{5}$ (\%): C, 72.62; H, 7.36; N, 11.68. Found: C, 72.51; H, 7.43; N, 11.54.

\section{Preparation of AOB-Y8 surfaces}

The weighed AOB-Y8 was mixed in a cap-sealed test vial with the specified ratio of $\mathrm{CHCl}_{3} / \mathrm{CH}_{3} \mathrm{CN}$ solvents. The mixture was heated until the solid dissolved, and then the sample vial was cooled to $35{ }^{\circ} \mathrm{C}$. The AOB-Y8 films were fabricated by casting a small drop of AOB-Y8 solution in $\mathrm{CHCl}_{3} / \mathrm{CH}_{3} \mathrm{CN}$ mixed solvents with different composition onto silicon plates. The silicon plates $(10 \mathrm{~mm} \times 10$ $\mathrm{mm}$ ) were cleaned by chloroform (corresponding water contact angle of $52.9^{\circ}$, Fig. S8 $\dagger$ ). For preparing the superhydrophobic AOB-Y8 surfaces with tuneable water adhesion, a certain volume $(100 \mu \mathrm{L})$ of AOB-Y8 solution with different concentrations in $50: 50 \mathrm{CHCl}_{3} / \mathrm{CH}_{3} \mathrm{CN}$ mixed solvents was casted onto the silicon plates. The evaporation rate of AOB-Y8 solution with $1 \mathrm{mg} \mathrm{mL}{ }^{-1}$, $2 \mathrm{mg} \mathrm{mL} \mathrm{mL}^{-1}, 3 \mathrm{mg} \mathrm{mL} \mathrm{m}^{-1}, 4 \mathrm{mg} \mathrm{mL} \mathrm{md}^{-1}$ and $\mathrm{mL}^{-1}$ is 7.69 $\mu \mathrm{L} \min ^{-1}, 8.45 \mu \mathrm{L} \min ^{-1}, 9.52 \mu \mathrm{L} \min ^{-1}, 10.52 \mu \mathrm{L} \min ^{-1}$ and

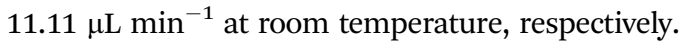

\section{Characterization}

All the reagents for the synthesis and spectral measurements were of spectroscopic grade and used as received. ${ }^{1} \mathrm{H}$ NMR spectra were recorded with a Bruker AVANCE III-300 $\mathrm{MHz}$ spectrometer, using tetramethylsilane (TMS) as an internal standard. Field emission scanning electron microscopy (FESEM) images were taken with a JSM-7001F apparatus for investigating the surface morphology of the as-prepared surfaces. The UV/Vis absorption spectra were carried out on a Cary 5000 UV-Vis-NIR Spectrophotometer. XRD experiments of samples were performed using a Bruker D8 advance diffractometer with $\mathrm{Cu}_{\mathrm{K} \alpha}$ radiation $(\lambda=1.54 \AA$, $40 \mathrm{kV}$, and $40 \mathrm{~mA})$. Data processing and analysis were carried out with Materials Data JADE (version 6) XRD pattern processing software.

The representative contact angle and sliding angle were measured using an optical contact angle meter DSA25S (KRUSS ADVANCE, Germany) with a water drop volume of $4 \mu \mathrm{L}$ at ambient temperature. The water contact angle of each surface was an average value calculated through at least four different points by means of sessile-drop method. A similar approach was explored to detect dynamic sliding angles (SAs), by means of slowly rotating the substrate with a $4 \mu \mathrm{L}$ water droplet until the water droplet began to slide. The contact angle hysteresis (CAH) was calculated from the difference between the values of advancing contact angle $\left(\theta_{\text {adv }}\right)$ and receding contact angle $\left(\theta_{\text {rec }}\right),{ }^{56}$ which were obtained by approaching and departing sample surface from settled sessile droplet until the contact line shifted, respectively. ${ }^{57,58}$

The corrosive water of different $\mathrm{pH}$ ranging from 1 to 14 was prepared by adding hydrochloric acid and/or sodium hydroxide. The adhesive forces of superhydrophobic surfaces were assessed through a high-sensitivity micro-electromechanical balance system (Dataphysics DCAT11, Germany). First, a water droplet of $5 \mu \mathrm{L}$ was suspended on a metal ring and the superhydrophobic surface was placed on the balance table. Then, the superhydrophobic surface was moved upward at a constant speed of $0.02 \mathrm{~mm} \mathrm{~s}^{-1}$ in an ambient environment with a relative humidity of about $35 \%$. Finally, the superhydrophobic surface was moved downward once in contact with the droplet while the balance force gradually increased until reached the maximum. The adhesive force was defined as the maximum force when the surface was just leaving the water droplet.

\section{Conclusions}

In summary, we have designed and synthesized a novel Yshaped compound AOB-Y8 consisting of azobenzene groups, 1,3,4-oxadiazole moieties, and three octyl chains, in order to regulate self-assembly structure in mixed solvents, and thus surface wettability and adhesion through solvent and concentration tuning. The surface hydrophobicity of AOB-Y8 films was successfully regulated by tuning the assembly morphologies through changing the polar interactions parameter of $\mathrm{CHCl}_{3}$ / $\mathrm{CH}_{3} \mathrm{CN}$ mixed solvents. By simply changing the concentration of AOB-Y8 in $\mathrm{CHCl}_{3} / \mathrm{CH}_{3} \mathrm{CN}$ solution, we have tuned the adhesion of superhydrophobic surface from a low level to a very high level. In the meantime, the self-assembled superhydrophobic surfaces of AOB-Y8 demonstrated an excellent chemical resistance to acid and alkali, suitable for applications in various environmental conditions. As examples, we have presented that the tuneable adhesive superhydrophobic AOB-Y8 surfaces can be effectively used in selective transportation of droplets, selfcleaning and drop-based microdetection or microreaction.

\section{Conflicts of interest}

There are no conflicts to declare. 


\section{Acknowledgements}

The authors are grateful to the National Science Foundation Committee of China (projects No. U1504510, U1604129, U1404619 and 21173068) for their financial support of this work.

\section{Notes and references}

1 K. Liu, X. Yao and L. Jiang, Chem. Soc. Rev., 2010, 39, 3240. 2 J. A. Howarter and J. P. Youngblood, Adv. Mater., 2007, 19, 3838. 3 J. Li, L. Yan, Q. Ouyang, F. Zha, Z. Jing, X. Li and Z. Lei, Chem. Eng. J., 2014, 246, 238.

4 J. Ou, W. Hu, M. Xue, F. Wang and W. Li, ACS Appl. Mater. Interfaces, 2013, 5, 3101.

5 S. Zhang, F. Lu, L. Tao, N. Liu, C. Gao, L. Feng and Y. Wei, ACS Appl. Mater. Interfaces, 2013, 5, 11971.

6 J. Li, X. Liu, Y. Ye, H. Zhou and J. Chen, Colloids Surf., A, 2011, 384, 109.

7 M. Liu and L. Jiang, Adv. Funct. Mater., 2010, 20, 3753.

8 J. Long, P. Fan, D. Gong, D. Jiang, H. Zhang, L. Li and M. Zhong, ACS Appl. Mater. Interfaces, 2015, 7, 9858.

9 J. Ju, K. Xiao, X. Yao, H. Bai and L. Jiang, Adv. Mater., 2013, 25, 5937.

10 R. Blossey, Nat. Mater., 2003, 2, 301.

11 M. H. Jin, X. J. Feng, L. Feng, T. L. Sun, J. Zhai, T. J. Li and L. Jiang, Adv. Mater., 2005, 17, 1977.

12 L. Feng, Y. A. Zhang, J. M. Xi, Y. Zhu, N. Wang, F. Xia and L. Jiang, Langmuir, 2008, 24, 4114.

13 J. Li, Z. Jing, F. Zha, Y. Yang, Q. Wang and Z. Lei, ACS Appl. Mater. Interfaces, 2014, 6, 8868.

14 E. Zhang, Y. Wang, T. Lv, L. Li, Z. Cheng and Y. Liu, Nanoscale, 2015, 7, 6151.

15 X. Mo, Y. Wu, J. Zhang, T. Hang and M. Li, Langmuir, 2015, 31, 10850.

16 D. Wu, S. Wu, Q. Chen, Y. Zhang, J. Yao, X. Yao, L. Niu, J. Wang, L. Jiang and H. Sun, Adv. Mater., 2011, 23, 545.

17 S. Boduroglu, M. Cetinkaya, W. J. Dressick, A. Singh and M. C. Demirel, Langmuir, 2007, 23, 11391.

18 Y. Lai, C. Lin, J. Huang, H. Zhuang, L. Sun and T. Nguyen, Langmuir, 2008, 24, 3867.

19 T. Darmanin and F. Guittard, Soft Matter, 2013, 9, 1500.

20 C. Li, R. Guo, X. Jiang, S. Hu, L. Li, X. Cao, H. Yang, Y. Song, Y. Ma and L. Jiang, Adv. Mater., 2009, 21, 4254.

21 M. Liu, Y. Zheng, J. Zhai and L. Jiang, Acc. Chem. Res., 2010, 43, 368.

22 E. Bormashenko, R. Pogreb, T. Stein, G. Whyman, M. Erlich, A. Musin, V. Machavariani and D. Aurbach, Phys. Chem. Chem. Phys., 2008, 10, 4056.

23 R. N. Wenzel, Ind. Eng. Chem., 1936, 28, 988.

24 A. B. D. Cassie and S. Baxter, Trans. Faraday Soc., 1944, 40, 546. 25 E. Bormashenko, R. Pogreb, G. Whyman and M. Erlich, Langmuir, 2007, 23, 12217.

26 X. Huang, D. Kim, M. Im, J. Lee, J. Yoon and Y. Choi, Small, 2009, 5, 90.

27 J. Yong, F. Chen, Q. Yang, D. Zhang, H. Bian, G. Du, J. Si, X. Meng and X. Hou, Langmuir, 2013, 29, 3274.
$28 \mathrm{~J}$. Ou, W. Hu, C. Li, Y. Wang, M. Xue, F. Wang and W. Li, ACS Appl. Mater. Interfaces, 2012, 4, 5737.

29 Z. Cheng, M. Du, H. Lai, N. Zhang and K. Sun, Nanoscale, 2013, 5, 2776.

30 B. A. Kakade, Nanoscale, 2013, 5, 7011.

31 X. Yu, Q. Zhong, H. Yang, L. Wan and Z. Xu, J. Phys. Chem. C, 2015, 119, 3667.

32 B. Zhou, J. Tian, C. Wang, Y. Gao and W. Wen, Appl. Surf. Sci., 2016, 389, 679.

33 H. Wang, Y. Zhu, Z. Hu, X. Zhang, S. Wu, R. Wang and Y. Zhu, Chem. Eng. J., 2016, 303, 37.

34 Y. Lu, S. Sathasivam, J. Song, C. R. Crick, C. J. Carmalt and I. P. Parkin, Science, 2015, 347, 1132.

35 W. Zhang, T. Xiang, F. Liu, M. Zhang, W. Gan, X. Zhai, X. Di, Y. Wang, G. Liu and C. Wang, ACS Appl. Mater. Interfaces, 2017, 9, 15776.

$36 \mathrm{H} . \mathrm{Wu}, \mathrm{K}$. Zhu, B. Cao, Z. Zhang, B. Wu, L. Liang, G. Chai and A. Liu, Soft Matter, 2017, 13, 2995.

37 Y. Liu, X. Li, J. Jin, J. Liu, Y. Yan, Z. Han and L. Ren, Appl. Surf. Sci., 2017, 400, 498.

38 J. Lv, Y. Song, L. Jiang and J. Wang, ACS Nano, 2014, 8, 3152. 39 S. B. Subramanyam, V. Kondrashov, J. Rühe and K. K. Varanasi, ACS Appl. Mater. Interfaces, 2016, 8, 12583.

40 M. Shimomura and T. Kunitake, J. Am. Chem. Soc., 1987, 109, 5175.

$41 \mathrm{H}$. Kobayashi, K. Koumoto, J. H. Jung and S. Shinkai, J. Chem. Soc., Perkin Trans. 1, 2002, 2, 1930.

42 S. Wang, L. Feng and L. Jiang, Adv. Mater., 2006, 18, 767.

43 Q. Zhang and J. Wang, Mater. Lett., 2012, 67, 334.

$44 \mathrm{~J}$. Li, X. Liu, Y. Ye, H. Zhou and J. Chen, J. Phys. Chem. C, 2011, 115, 4726.

45 C. M. Hansen, Hansen Solubility Parameters: A User's Handbook, CRC Press LLC, Boca Raton, FL, 2nd edn, 2007;

C. M. Hansen, Prog. Org. Coat., 2004, 51, 77.

46 A. A. Abdala, K. Olesen and S. A. Khan, J. Rheol., 2003, 47, 497. 47 H. Xu, J. Song, T. Tian and R. Feng, Soft Matter, 2012, 8, 3478.

48 J. X. Cui, J. Zheng, W. Q. Qiao and X. H. Wan, J. Colloid Interface Sci., 2008, 326, 267.

49 L. B. Niu, J. Song, J. J. Li, N. M. Tao, M. Lu and K. Q. Fan, Soft Matter, 2013, 9, 7780.

50 X. Yao, J. Gao, Y. Song and L. Jiang, Adv. Funct. Mater., 2011, 21, 4270.

51 Z. Cheng, R. Hou, Y. Du, H. Lai, K. Fu, N. Zhang and K. Sun, ACS Appl. Mater. Interfaces, 2013, 5, 8753.

52 H. Song, D. L. Chen and R. F. Ismagilov, Angew. Chem., Int. $E d .$, 2006, 45, 7336.

53 J. Liu and Y. Chiu, J. Polym. Sci., Part A: Polym. Chem., 2010, 48, 1142. 54 Y. Zhang, K. G. Jespersen, M. Kempe, J. A. Kornfield, S. Barlow, B. Kippelen and S. R. Marder, Langmuir, 2003, 19, 6534 .

55 D. Zha and L. You, ACS Appl. Mater. Interfaces, 2016, 8, 2399. 56 D. Jeong, S. Kim, J. Park, S. Kim, D. Lee and J. Kim, ACS Appl. Mater. Interfaces, 2014, 6, 2770.

57 Y. Xue, Y. Wu, X. Pei, H. Duan, Q. Xue and F. Zhou, Langmuir, 2015, 31, 226.

58 Y. Wu, Y. Xue, X. Pei, M. Cai, H. Duan, W. T. S. Huck, F. Zhou and Q. Xue, J. Phys. Chem. C, 2014, 118, 2564. 